\title{
Eficácia da medida de antígeno prostático específico para rastreamento de carcinoma de próstata e seus impactos na saúde do homem
}

\author{
Effectiveness of prostate-specific antigen measurement for prostate cancer screening and \\ its impacts on men's health
}
Efectividad de la medición del antígeno prostático específico para la detección del cáncer de próstata y sus impactos en la salud de los hombres

Emanuelle Ferrão Lopes $^{1 *}$, Marina Souza Scholant ${ }^{1}$, Laura Böttcher Lins ${ }^{1}$, Juliana Dick Casagrande $^{1}$, Camila Horst da Silva ${ }^{1}$, Shanady Mahmud Khaled ${ }^{1}$, João Gabriel Duarte Siqueira ${ }^{1}$.

\section{RESUMO}

Objetivo: Revisar estudos prévios que tratam da efetividade da medida do Antígeno Prostático Específico (PSA) para o diagnóstico de carcinoma prostático, a fim de entender a necessidade do rastreamento na população-alvo, e expor as implicações do resultado positivo da dosagem dessa protease. Revisão bibliográfica: A utilização da dosagem sérica de PSA se mostrou eficaz para detecção das principais patologias prostáticas, mas ineficiente quando utilizado isoladamente para diferenciação entre as patologias. Assim, fazem-se necessários o uso de exames combinados, como o toque retal e a densidade do PSA, a fim de acrescentar clinicamente. Ademais, o uso exclusivo da dosagem de PSA e sua utilização fora da população-alvo estabelecida pelos estudos acarretam consequências sérias na saúde do homem em casos de resultados positivos. Considerações finais: Diante das divergências existentes na indicação do rastreamento do câncer de próstata e a constante atualização dos dados referentes à esta, é dever do profissional da saúde manter-se atualizado, visando à aplicação do exame de forma correta e bem indicada. Além disso, deve estar preparado para realizar o manejo dos impactos gerados a partir da ampla utilização da medida do PSA, a fim de garantir a segurança do paciente em todos os aspectos.

Palavras-chave: Antígeno prostático específico, Câncer de próstata, Rastreamento.

\begin{abstract}
Objective: To review previous studies dealing with the effectiveness of the measurement of the Specific Prostatic Antigen (PSA) for the diagnosis of prostate cancer, in order to understand the need for screening in the target population, and to expose the implications of the positive result of the dosage of this protease. Bibliographic review: The use of serum PSA dosage proved to be effective in detecting the main prostatic diseases, but inefficient when used alone to differentiate between pathologies. Thus, it is necessary to use combined tests, such as digital rectal examination and PSA density. Moreover, the exclusive use of PSA dosage and its use outside the target population established by the studies have serious consequences for men's health in cases of positive results. Final considerations: Given the divergences in the indication for prostate cancer screening and the constant updating of data related to it, it is the duty of the health professional to keep up to date, aiming at the application of the test in a correct and well-applied way. In addition, it must be prepared to manage the impacts generated from the wide use of the PSA measurement, in order to ensure patient safety in all aspects.
\end{abstract}

Keywords: Prostate-specific antigen, Prostate cancer, Screening.

1 Universidade Católica de Pelotas (UCPel), Pelotas - RS. *E-mail: emanuelle.ferrao@sou.ucpel.edu.br 


\section{RESUMEN}

Objetivo: Revisar los estudios previos sobre la eficacia de la medición del Antígeno Prostático Específico (PSA) para el diagnóstico del carcinoma de próstata, con el fin de comprender la necesidad del cribado en la población diana, y exponer las implicaciones del resultado positivo de la dosificación de esta proteasa. Revisión bibliográfica: El uso del PSA en suero ha demostrado ser eficaz para la detección de las principales enfermedades prostáticas, pero es ineficaz cuando se utiliza solo para diferenciar las enfermedades. Por ello, es necesario utilizar pruebas combinadas, como el tacto rectal y la densidad del PSA, para agregar clínicamente. Además, el uso exclusivo de la dosis de PSA y su utilización fuera de la población objetivo establecida por los estudios conlleva graves consecuencias para la salud de los hombres en caso de resultados positivos. Consideraciones finales: Dadas las diferencias existentes en la indicación del cribado del cáncer de próstata y la constante actualización de datos al respecto, es deber de los profesionales sanitarios mantenerse al día, con el objetivo de aplicar la prueba de forma correcta y bien indicada. Además, deben estar preparados para gestionar los impactos generados por el uso generalizado de la medición de la PSA, con el fin de garantizar la seguridad del paciente en todos los aspectos.

Palabras clave: Antígeno prostático específico, Cáncer de próstata, Cribado.

\section{INTRODUÇÃO}

O Antígeno Prostático Específico (PSA) é uma protease encontrada no epitélio da próstata e excretada no líquido seminal, que tem a função de tornar o fluido liquefeito, por isso, sua concentração plasmática deve ser pequena quando em condições normais (JUNIOR A, et al., 2010). Ainda segundo Junior A, et al. (2010), é possível afirmar que a dosagem desta enzima se tornou um método de rastreio importante para o câncer de próstata e traz benefícios para o diagnóstico precoce dessa neoplasia e redução da morbimortalidade associada. Por outro lado, o uso difundido desse rastreamento levanta debates acerca da ocorrência de diagnósticos excessivos e tratamentos desnecessários, que podem afetar a saúde mental masculina e acarretar potenciais danos iatrogênicos.

O aumento da expectativa de vida e o envelhecimento da população levaram a necessidade de evidenciar cientificamente métodos para a detecção precoce de doenças crônicas não transmissíveis, uma vez que essas aumentam significativamente desde as últimas décadas do século XX, o que se tornou um desafio para a saúde, especialmente para os sistemas públicos e universais. Dentre essas doenças, destacamos o câncer, em suas diversas apresentações clínicas e graus de gravidade, que repercutem na saúde e na qualidade de vida da população (INSTITUTO NACIONAL DO CÂNCER (INCA), 2020).

Indubitavelmente, ter conhecimento acerca dos testes diagnósticos é essencial e importante no que tange ao rastreamento dessa doença, pois é necessário assegurar que haja mais vantagens que prejuízos. Dado isso, o método de rastreio deve ser baseado em evidências científicas, a fim de maximizar os benefícios com a detecção precoce e reduzir os danos ao paciente (STEFFEN RE, et al., 2018).

Nesse contexto, o câncer se caracteriza pela multiplicação rápida e desorganizada de células, levando à formação de um tumor capaz de enviar metástases, gerar inúmeros sintomas sistêmicos e, no pior cenário, levar à morte (SARRIS AB, et al., 2018). No entanto, quando se trata da próstata, de forma geral, o câncer se desenvolve inicialmente de forma assintomática e cresce de maneira lenta, muitas vezes não oferecendo prejuízos à saúde do homem por toda a sua vida (STEFFEN RE, et al., 2018). A partir disso, foram determinados critérios importantes para avaliar a necessidade ou não de rastreamento do câncer prostático, dentre eles, o histórico familiar, fatores alimentares e, em destaque, a idade, visto que a patologia tem sua incidência aumentada em homens acima de 50 anos (CZORNY RN, et al., 2017).

De acordo com o INCA, o câncer de próstata representa $29 \%$ das neoplasias malignas no país, o que aponta para uma perspectiva de 65.840 novos casos por ano entre 2020 e 2022, demonstrando a importância do debate desse assunto sob uma perspectiva social. A crescente incidência da doença reforça a necessidade do questionamento acerca do aumento no número de diagnósticos e do início de tratamentos agressivos com potencial dano para a saúde do homem (HOFFMAN RM, 2021). 
Desse modo, o objetivo deste artigo foi pontuar o quão eficaz é a medida do PSA para o diagnóstico do carcinoma prostático e quais são as proporções de benefício e malefício do rastreamento da doença por meio dessa protease.

\section{REVISÃO BIBLIOGRÁFICA}

\section{Utilização do rastreamento}

A detecção precoce do câncer prostático, bem como dos demais carcinomas, pode ser realizada por meio de duas maneiras: a primeira refere-se ao rastreamento, que tem por objetivo encontrar diagnósticos de câncer em pacientes sem sintomas ou a presença de lesões pré-cancerígenas nestes mesmos pacientes, por meio de exames de rotina. A segunda forma é o diagnóstico precoce, que busca identificar a presença do carcinoma em estágios primários, naqueles pacientes que apresentam algum indicativo (sinais e sintomas) que levem a suspeitar da doença (CZORNY RN, et al., 2017).

Segundo a Sociedade Americana de Cancerologia (2020), é indicado a triagem em homens assintomáticos a partir dos 50 anos de idade, através da dosagem do Antígeno Prostático Específico (PSA), acompanhado do toque retal. Indica-se, assim, que o PSA tem um valor significativamente maior quando acompanhado do exame de toque retal. Damião $\mathrm{R}$, et al. (2015) afirma que pesquisas em que se utilizaram da associação dos dois exames, toque e PSA, verificou-se que $18 \%$ dos tumores não seriam identificados sem utilizar-se do toque retal. Czorny RN, et al. (2017) destaca, ainda, que o exame de toque retal é capaz de conseguir estimar o volume prostático, sua consistência e, assim, a possibilidade de estar acometido por neoplasia.

Conforme resultados de um estudo europeu randomizado de triagem de câncer de próstata (European Randomized Screening for Prostate Cancer Trial), realizado durante 13 anos, há redução substancial de mortalidade por câncer de próstata em 9-10 anos de rastreamento com dosagem de PSA. Esse estudo foi um ensaio multicêntrico randomizado que, por sua vez, avaliou o rastreamento com dosagem de PSA em oito países europeus, baseado em um banco de dados pré-definido e uma população de idade média de 55 a 69 anos. Ainda assim, esse tipo de abordagem é controverso pelos seus efeitos adversos, como por exemplo, aumento de sobrediagnósticos e medidas invasivas desnecessárias (BAGCCHI S, 2014).

\section{Barreiras no rastreamento pelo PSA e toque retal}

Um estudo transversal, descritivo, de caráter quantitativo feito por Menezes R, et al. (2019) foi realizado com 130 homens, acima de 40 anos e demonstrou que embora os homens reconheçam que têm necessidades em saúde, muitas vezes retardam e hesitam em procurar os serviços de apoio, ou seja, possuem uma falha na adesão às medidas de autocuidado e prevenção. Essas ações muitas vezes são encorajadas por variáveis culturais, pois se veem fortes e com a saúde invulnerável.

De acordo com Leite de Moraes M, et al. (2017) o maior desafio na detecção precoce do câncer de próstata seja a resistência dos homens em realizar o toque retal, pois acredita-se que exista uma dificuldade dos homens em aceitar suas dificuldades e o envolvimento de questões culturais, como medo, preconceito, vergonha e a falta de coragem para realizar os exames. Com isso, exames como toque retal e o PSA, os quais possuem suma importância para diagnóstico de câncer de próstata, muitas vezes não são realizados por esses motivos, fato esse que prejudica o rastreamento do câncer de próstata, tanto em seu início quanto, até mesmo, em estado mais avançado. Além disso, os homens acabam por procurar os serviços médicos quando as dores já estão insuportáveis, ou que já afetou o seu rendimento no trabalho. Fato que prejudica o diagnóstico no estágio inicial.

\section{Efetividade do rastreamento e consequências de um resultado positivo}

Análises que puseram em pauta a eficácia do rastreamento do câncer de próstata com PSA alegam que a taxa de resultados falso-positivos varia de acordo com o limiar do resultado de 11,3\% (quando o limiar utilizado é de 4,0 ng/ml) a 19,8\% (quando é de 3,0 ng/ml) (SCHRÖDER FH, et al., 2014). Os pacientes que têm resultado positivo seguem, na maioria das vezes, para a investigação diagnóstica com biópsia de próstata (FENTON JJ, et al., 2018). 
Nos casos de anormalidade do PSA e resultados benignos na biópsia, a preocupação com o câncer de próstata foi consideravelmente maior quando comparada aos que não tiveram resultados anormais no rastreamento. Esse sentimento foi relatado por todos rastreados que obtiveram esse tipo de resultado, sem embargo de normalidade na biópsia (UNITED STATES PREVENTIVE SERVICES TASK FORCE, 2018). A partir dessas evidências, cada vez mais os órgãos de saúde internacionais trazem à tona a necessidade de prévio debate sobre os benefícios e os malefícios dessa abordagem e a necessidade de individualizar e avaliar o performance status do paciente, uma vez que essa é potencial causadora de danos (STEFFEN RE, et al., 2018).

De acordo com Schröder FH, et al. (2014), o estudo europeu randomizado sobre triagem de câncer de próstata demonstrou resultados significativos na redução da mortalidade da doença após 9 a 13 anos de seguimento dos pacientes. Entretanto, o mesmo estudo pontua que 40 a $50 \%$ dos casos identificados tratavam-se de sobrediagnósticos, que causam danos evitáveis capazes de afetar diretamente a saúde do homem.

Cabe pontuar que existem prejuízos nesse rastreamento do câncer de próstata, como danos próprios do teste de PSA e danos encontrados subsequentemente ao diagnóstico e ao tratamento. De acordo com a United States Preventive Services Task Force (2018), existem malefícios acerca do rastreio por teste PSA, como resultados falso-positivos, que acarretam consequências nocivas à saúde psicológica do paciente, sobrediagnósticos e a identificação de câncer assintomático que nunca causaria dano ou contribuiria para a morte, classificando a evidência cientifica para o rastreamento do câncer de próstata como do tipo $D$. Ademais, quando necessários outros testes além do PSA e toque retal por dúvida diagnóstica, é indicada a realização de biópsia, a qual pode ocasionar episódios de dor, hematospermia e infecções.

A fim de demonstrar a discordância entre as diretrizes, o INCA (2020), afirma que os estudos já feitos não são suficientes a fim de pontuar se realmente há diminuição da mortalidade por câncer com o rastreamento pelo toque retal e/ou dosagem do PSA. Por outro lado, a American Cancer Society (2019) postula que essas medidas devem ser realizadas com uma periodicidade anual em homens com idade igual ou superior a 50 anos e com uma expectativa de vida de pelo menos 10 anos e, ainda, que os homens pertencentes aos grupos de risco devem iniciar o rastreio aos 45 anos.

Segundo Duffy MJ (2011), o PSA é classificado como um marcador falho no rastreamento de câncer de próstata em pacientes assintomáticos, pelo seu potencial de superdetecção e, consequentemente, tratamento desnecessário ou inadequado para homens com diagnóstico positivo. Fundamenta-se que o PSA não apresenta um ponto de corte que classifica homens com presença ou ausência de células malignas na amostra. Dessa forma, doenças benignas, como Hiperplasia Prostática Benigna (HPB), prostatite ou, até mesmo, obstrução urinária, podem apresentar níveis séricos de PSA elevados (DUFFY MJ, 2011).

\section{Faixa etária estabelecida de rastreamento}

O estudo realizado por Heck J, et al. (2013) concluiu que a incidência de valores elevados de Antígeno Prostático Específico ocorre com maior frequência no grupo de homens maiores de 70 anos, o que corrobora com a faixa etária estabelecida para o rastreamento. Além disso, esse estudo pontua que dentre os pacientes da amostra que possuíam PSA aumentado, a maioria se tratava de diagnósticos de Hiperplasia Prostática Benigna. Ainda assim, Heck J, et al. (2013) pontua a necessidade de triar esses homens que estão dentro da faixa de risco para doenças prostáticas, ao menos para afastar a possibilidade de neoplasia.

De acordo com uma análise transversal em banco de dados feito por Mori RR, et al. (2020), foram separados homens em dois grupos de faixas etárias, o grupo A com 45-69 anos e grupo B $\geq 70$ anos, em que houve uma comparação de prevalência de câncer de próstata por meio da análise de resultados como teste de PSA prévios e critérios de agressividade. Sendo assim, foi visto que os níveis médios de PSA foram maiores no grupo $B$, a prevalência de câncer de próstata foi maior no grupo $B$ e as chances de doença de risco intermediário/alto também vão de encontro com essa faixa etária. Nesse sentido, devido aos achados desse estudo, concluiu-se que a faixa etária de rastreamento de câncer de próstata é de 70 anos ou mais, com expectativa de vida de, no mínimo, 10 anos, o que corrobora com o estudo de Heck J, et al. (2013). 


\section{Utilização da medida do PSA total e do PSA livre}

O Antígeno Prostático Específico da corrente circulatória, quando dosado de forma total, considera a porção do antígeno ligada a proteínas, somada à porção livre. De acordo com Souza $F$ (2015), as células prostáticas malignas não produzem maior quantidade de PSA do que as benignas, entretanto, as malignas que são capazes de produzir PSA possuem propensão a ligarem-se às proteínas plasmáticas. Dessa forma, pacientes com câncer são suscetíveis a terem níveis reduzidos da porção livre do PSA, o que justifica a dosagem dessa porção da enzima.

\section{Medida da densidade em comparação ao PSA isolado}

Em consonância com o fato de que a medida do PSA de forma isolada aumenta significativamente as taxas de sobrediagnóstico e tratamentos com potenciais danos iatrogênicos, o estudo conduzido por Nordström T, et al. (2017) utilizou a medida da densidade do antígeno prostático específico, calculada pela razão entre o PSA total $(\mathrm{ng} / \mathrm{ml})$ e volume da próstata $(\mathrm{ml})$ como método comparativo ao exame PSA isolado para rastreamento do câncer de próstata clinicamente significativo.

Nordström T, et al. (2017) concluiu que a medida da densidade do PSA, utilizando-se da medição do volume prostático através de ultrassom transrretal e ressonância magnética, é capaz de rastrear casos de câncer de próstata clinicamente significativos de forma eficiente, principalmente quando associado às informações clínicas, como histórico familiar, história patológica pregressa e exposição a fatores de risco.

De acordo com Joshua SJ, et al. (2017), o cálculo da densidade de PSA é mais eficaz que a dosagem de PSA para diagnóstico de qualquer câncer de próstata em homens com ou sem biópsia. O estudo foi realizado com 1290 pacientes, destes, 585 com câncer de próstata e 284 com câncer de próstata clinicamente significativo. Neles, a densidade prostática se mostrou eficaz para a diferenciação dos casos, em faixas de dosagem de PSA entre $4-10 \mathrm{ng} / \mathrm{ml}$ e $>10 \mathrm{ng} / \mathrm{ml}$ (JOSHUA SJ, et al., 2017).

Por outro lado, o estudo realizado por Czorny RN, et al. (2017) pontua que o cálculo da densidade do PSA necessita de mais estudos, porém, pode ser ainda associado à velocidade do PSA, ou seja, o acréscimo ou decréscimo do antígeno ao longo do tempo, o que auxilia ainda mais e com mais bases científicas a determinação da probabilidade do carcinoma de próstata.

\section{Medida do p2PSA e cálculo do índice de saúde da próstata (PHI)}

São descritas 4 isoformas do Antígeno Prostático Específico, p2PSA, p4PSA, p5PSA e p7PSA, de acordo com a cadeia de peptídeos. Dentre esses, o p2PSA é o mais específico marcador para câncer de próstata e é a partir dele que o cálculo do PHI é realizado, por meio de uma fórmula matemática ([p2PSA/PSA livre] $x$ VPSA total) (CHENG YT, et al., 2019). De acordo com Cheng YT, et al. (2020), a utilização da dosagem de p2PSA aumenta a sensibilidade de rastreio do câncer de próstata, além de possuir um melhor valor preditivo.

Ainda segundo Cheng YT, et al. (2020), a utilização do cálculo do PHI foi aprovada em 2012 pela Food and Drug Administration (FDA) para detecção do carcinoma prostático quando os valores séricos de PSA total estiverem entre 4 e $10 \mathrm{ng} / \mathrm{ml}$, podendo estes valores de corte variarem de acordo com a etnia do paciente. Ainda não existe um consenso entre a literatura sobre os níveis exatos de PHI e p2PSA, entretanto é de comum acordo que esta fração do PSA é a mais sensível e específica para o câncer, se sobrepondo à dosagem de PSA total/PSA livre (CHENG YT, et al., 2019).

\section{Paralelo entre hiperplasia prostática benigna e câncer de próstata}

Em relação à clínica das principais patologias da próstata, e câncer, a HPB se caracteriza por sintomas de obstrução uretral, como redução da força do jato urinário e esvaziamento incompleto da bexiga. O câncer, por outro lado, costuma se apresentar de forma assintomática em seu início e, posteriormente, pode cursar com sintomas semelhantes à hiperplasia e, por fim, pode dar sintomas secundários à formação de metástases (DAMIÃO R, et al., 2015)

De acordo com um estudo realizado por Kim J, et al. (2015), a flutuação do PSA é significativamente maior em pacientes sem câncer de próstata do que em pacientes com a doença. Como foi observado no estudo, 
$25 \%$ dos homens com níveis de PSA de 4 a $10 \mathrm{ng} / \mathrm{ml}$ apresentaram câncer de próstata comprovado por biópsia. Porém, $75 \%$ foram submetidos a biópsias de próstata desnecessárias, as quais poderiam causar ansiedade, desconforto e custos adicionais significativos com cuidados de saúde. Além disso, o estudo concluiu que o benefício do rastreamento permanece controverso, porém pode ser valioso ao ser usado com outros índices de PSA, como o PSA livre.

Um estudo transversal realizado em homens com valores de PSA entre 2,6 e 10,0 ng/ml, demonstrou que a dosagem da protease prostática aumenta a detecção do câncer em até $81 \%$ quando comparado com o uso do toque retal isolado. Além disso, essa mesma análise identificou que os volumes prostáticos de pacientes com câncer eram em média de $41,4 \mathrm{~cm}^{3}$ menores do que nos pacientes com resultado negativos, que correspondiam a 59,9 $\mathrm{cm}^{3}$ (CASTRO HAS, et al., 2011).

Para diferenciar a HPB do câncer de próstata utiliza-se a medida do PSA, antes da realização de qualquer procedimento. Em consonância com essa afirmação, um estudo realizado por Strittmatter $F$, et al. (2011) analisou dois grupos: homens com HPB e homens com câncer de próstata. A partir disso, o objetivo do estudo é avaliar o desempenho dos seguintes marcadores: PSA total (tPSA), razão entre PSA livre e PSA total (f/t PSA), PSA complexado (cPSA) e razão entre PSA complexado e PSA total (c/t PSA).

Ao serem analisados os resultados do estudo de Strirrmatter $F$, et al. (2011), foi observado que, para valores de tPSA de até $4 \mathrm{ng} / \mathrm{mL}$, a medida de tPSA apresentou sensibilidade de $82 \%$ e especificidade de $58 \%$, e o f/t PSA sem apresentar benefícios adicionais. Quando o tPSA estava entre $4-10 \mathrm{ng} / \mathrm{mL}$, o melhor poder de discriminação entre HPB e câncer de próstata é o marcador f/t PSA, com sensibilidade de $85 \%$ e especificidade de $58 \%$. Por fim, em intervalos de tPSA $>10 \mathrm{ng} / \mathrm{mL}$, o cPSA apresentou uma capacidade de diferenciação maior entre os grupos.

Nesse contexto, como já foi citado no presente trabalho, é essencial que se evite prejuízos ao paciente ao realizar o rastreamento de câncer de próstata, principalmente utilizando métodos que possam ajudar o diagnóstico ao diferenciar doenças que alteram o PSA além do câncer, como a HPB (STEFFEN RE, et al., 2018).

\section{CONSIDERAÇÕES FINAIS}

A medida do PSA com intuito de rastrear patologias prostáticas é especialmente relevante em homens acima dos 70 anos, considerando-se que a extensa classe de doenças desse órgão surge nesta faixa etária. Assim sendo, é necessário que esta parcela populacional esteja ciente da importância da realização do exame, ainda que nem todo resultado positivo na dosagem sérica de PSA seja indicativo de câncer. Acima disso, cabe ao profissional da saúde manter-se atualizado sobre os exames complementares ao diagnóstico do carcinoma prostático a fim de proporcionar a indicação correta para o paciente e minimizar os potenciais danos de um sobrediagnóstico.

\section{REFERÊNCIAS}

1. AMERICAN CANCER SOCIETY (ACS). Prostate Cancer. 2019. Disponível em: https://www.cancer.org/content/dam/CRC/PDF/Public/8795.00.pdf. Acessado em: 19 de jul. de 2021.

2. ARAÚJO FR, et al. Evaluation of PSA requests in men under 40 years of age. Jornal Brasileiro de Patologia e Medicina Laboratorial, 2020; 56: 1-5.

3. BAGCCHI S. PSA testing beneficial for prostate cancer. Lancet Oncology, 2014; 15(10): e424

4. CASTRO HAS, et al. Contribuição da densidade do PSA para predizer o câncer de próstata em pacientes com valores de PSA entre 2,6 e $10 \mathrm{ng} / \mathrm{ml}$. Radiol. Bras., 2011; 44(4): 205-209.

5. CHENG YT, et al. Preoperative \%p2PSA and Prostate Health Index Predict Pathological Outcomes in Patients with Prostate Cancer Undergoing Radical Prostatectomy. Sci Rep, 2020; 10(1): 776.

6. CHENG YT, et al. The application of p2PSA\% and prostate health index in prostate cancer detection: A prospective cohort in a Tertiary Medical Center. J Formos Med Assoc., 2019; 118(1;2): 260-267.

7. CZORNY RN, et al. Fatores de risco para o câncer de próstata: população de uma Unidade Básica de Saúde. Cogitare Enfermagem, 2017; 22(4): e51823v. 
8. DAMIÃO R, et al. Câncer de próstata. Revista Hospital Universitário Pedro Ernesto, 2015; 14: 80-86

9. DUFFY MJ. Prostate -specific antigen: does the current evidence support its use in prostate cancer screening? Ann Clin Biochem, 2011; 48(4): 310-6.

10. FENTON JJ, et al. Prostate-Specific Antigen-Based Screening for Prostate Cancer: Evidence Report and Systematic Review for the US Preventive Services Task Force. JAMA. 2018; 319(18): 1914-1931

11. HECK J, et al. Avaliação do Nível Sérico de Antígeno Prostático Específico (PSA) e Relação com Hiperplasia Benigna Prostática e Câncer de Próstata em Pacientes Atendidos em um Laboratório de Análises Clínicas. Acta Biomedica Brasiliensia, 2013; 4(1).

12. HOFFMAN RM. Screening for prostate cancer. N Engl J Med 2011; 365: 2013-2019.

13. INSTITUTO NACIONAL DO CÂNCER (INCA). Câncer de Próstata, 2021. Disponível em: https://www.inca.gov.br/tipos-de-cancer/cancer-de-prostata/profissional-de-saude. Acessado em: 28 de ago. de 2021.

14. JOSHUA SJ, et al. ReExamining PSA Density: Defining the Optimal PSA Range and Patients for Using PSA Density to Predict Prostate Cancer Using Extended Template Biopsy. Urology. 2017; 105: 123-128

15. JUNIOR A, et al. Urologia fundamental. Sociedade Brasileira de Urologia. 1a ed. São Paulo: Editora Planark, 2010; 189-194p.

16. KIM JS, et al. Prostate-Specific Antigen fluctuation: what does it mean in diagnosis of prostate cancer?. Revista Eletrônica International Braz J Urol, 2015; 41(2): 258-264

17. LEITE DE MORAES M, et al. Uma questão masculina: conhecendo possíveis entraves para a realização dos exames de detecção do câncer de próstata. Revista Medica Herediana. 2017; 4: 230-235p.

18. MENEZES R, et al. Conhecimento, Comportamento e Práticas em Saúde do Homem em Relação ao Câncer de Próstata. Rev Fund Care Online, 2019; 11(5): 1173-1179.

19. MORI RR, et al. Prostate cancer screening among elderly men in Brazil: should we diagnose or not? Revista Eletrônica International Braz J Urol, 2020; 46(1): 34-41.

20. NORDSTRÖM T, et al. Prostate-specific antigen (PSA) density in the diagnostic algorithm of prostate cancer. Prostate Cancer and Prostatic Diseases, 2018; 21(1): 57-63.

21. SARRIS AB, et al. Câncer de próstata: uma breve revisão atualizada. Visão Acadêmica, 2018; 19(1).

22. SCHRÖDER FH, et al. Screening and prostate cancer mortality: results of the European Randomised Study of Screening for Prostate Cancer (ERSPC) at 13 years of follow-up. The Lancet, 2014; 384(9959): 2027-35.

23. STEFFEN RE, et al. Rastreamento populacional para o câncer de próstata: mais riscos que benefícios. Physis: Revista de Saúde Coletiva, 2018; 28(2): e280209.

24. STRITTMATTER F, et al. Detection of prostate cancer with complexed PSA and complexed/total PSA ratio - is there any advantage?. European journal of medical research, 2011; 16(10): 445-50.

25. UNITED STATES PREVENTIVE SERVICES TASK FORCE. Recommendation Prostate Cancer: Screening. 2018. Disponível em: https://www.uspreventiveservicestaskforce.org/uspstf/recommendation/prostate-cancer-screening. Acessado em: 19 de jul. de 2021. 